J. Korean Math. Soc. 48 (2011), No. 5, pp. 969-984

http://dx.doi.org/10.4134/JKMS.2011.48.5.969

\title{
HYPERCYCLICITY OF WEIGHTED COMPOSITION OPERATORS ON THE UNIT BALL OF $\mathbb{C}^{N}$
}

\author{
Ren-Yu Chen and Ze-Hua Zhou
}

\begin{abstract}
This paper discusses the hypercyclicity of weighted composition operators acting on the space of holomorphic functions on the open unit ball $B_{N}$ of $\mathbb{C}^{N}$. Several analytic properties of linear fractional selfmaps of $B_{N}$ are given. According to these properties, a few necessary conditions for a weighted composition operator to be hypercyclic in the space of holomorphic functions are proved. Besides, the hypercyclicity of adjoint of weighted composition operators are studied in this paper.
\end{abstract}

\section{Introduction}

Let $B_{N}$ be the open unit ball in $\mathbb{C}^{N}$. Each holomorphic self-map $\varphi$ on $B_{N}$ is associated with a linear operator $C_{\varphi}$, the composition operator with symbol $\varphi$, which is given by the rule $C_{\varphi}(f)=f \circ \varphi$ for every $f \in H\left(B_{N}\right)$. Here $H\left(B_{N}\right)$ denotes the space consisting of all holomorphic functions on $B_{N}$. The multiplication operator $M_{\psi}$ induced by $\psi \in H\left(B_{N}\right)$ is defined by $M_{\psi}(f)=\psi \cdot f$. The weighted composition operator $W_{\psi, \varphi}$ with symbols $\varphi$ and $\psi$ is defined to be the linear operator on $H\left(B_{N}\right)$ with $W_{\psi, \varphi}=M_{\psi} C_{\varphi}$. It should be noted that $H\left(B_{N}\right)$ is an F-space when endowed with the metric where a sequence $\left\{f_{n}\right\}$ in $H\left(B_{N}\right)$ converges to $f \in H\left(B_{N}\right)$ if and only if $f_{n} \rightarrow f$ in the sense of compact-open topology.

According to the definition of [22], for any $w \in \partial B_{N}$ and any positive number $\alpha, \operatorname{Lip}_{\alpha}(w)$ corresponds to the class of holomorphic functions $\psi$ with domain $B_{N}$ such that there is some neighborhood $G$ for $w$ and a positive constant $M$ with

$$
|\psi(z)-\psi(w)| \leq M|z-w|^{\alpha} \text { for } z \in G \cap B_{N} .
$$

Let $X$ be an F-space and $T$ be a bounded linear operator on $X$. For $x \in$ $X$, let $\operatorname{Orb}(T, x)=\left\{T^{n} x: n \in \mathbb{N}\right\}$ be an orbit of $T$. Then $T$ is said to be

Received February 26, 2010; Revised January 26, 2011.

2010 Mathematics Subject Classification. Primary 47B33; Secondary 47B35, 47B38, 46E15, 32A 36.

Key words and phrases. hypercyclic operator, weighted composition operator, linear fractional map, generalized Cayley transform, Heisenberg transform, Denjoy-Wollf point.

Supported in part by the National Natural Science Foundation of China (Grand Nos. 10971153, 10671141). 
hypercyclic if the orbit $\operatorname{Orb}(T, x)$ is dense in $X$ for some $x \in X$. In this case, $x$ is called a hypercyclic vector for $T$. The hypercyclicity of composition operators in one complex variable has been discussed by Bourdon and Shapiro in $[3,4]$. For composition operators with linear fractional symbols on $H^{2}\left(B_{N}\right)$, some results have been obtained in $[1,2,7,16]$. And for weighted composition operators in one dimensional case, B. Yousefi and H. Rezaei [22] showed that the hypercyclicity of $W_{\psi, \varphi}$ depended on how freely $\psi(z)$ was allowed to approach the unit circle as $z \rightarrow w$, where $w$ was the Denjoy-Wolff point of $\varphi$. Some other types of hypercyclic operators can be found in $[6,11,12,14,15,19,21]$.

In this paper, $\left\{\varphi_{n}\right\}$ denotes the iteration sequence of $\varphi$. B. Yousefi and H. Rezaei [22] pointed out that if $\varphi$ is a self-map of the unit disk of $\mathbb{C}$, $\sum_{n=1}^{\infty}\left(1-\left|\varphi_{n}(z)\right|\right)^{\alpha}$ converges uniformly on compact subset of the unit disk whenever $\varphi$ is hyperbolic and $\alpha>0$ or $\varphi$ is a parabolic automorphism and $\alpha>\frac{1}{2}$. In higher dimensional cases, the situation is much more complicated. We will show that the series $\sum_{n=1}^{\infty}\left(1-\left|\varphi_{n}(z)\right|^{2}\right)^{\alpha}$ converges if one of the following cases holds: $\varphi$ is a hyperbolic self-map of $B_{N}$ and $\alpha>0 ; \varphi$ is a parabolic liner fractional map with at least one invariant slice and $\alpha>1 ; \varphi$ is a parabolic linear fractional map and the restriction of $\varphi$ on one of its invariant slices is an automorphism and $\alpha>1 / 2 ; \varphi$ is a parabolic automorphism without invariant slices and $\alpha>1 / 4$. As a consequence, under the additional hypotheses that $\psi \in \operatorname{Lip}_{2 \alpha}(w)$ and $\psi$ never vanishes in $B_{N}$, an eigenvalue and the corresponding eigenvector for $W_{\psi, \varphi}$ are calculated. Moreover, due to Theorem 4.1, $W_{\psi, \varphi}$ is hypercyclic if $C_{\varphi}$ is hypercyclic on $H\left(B_{N}\right)$, where $\varphi$ and $\psi$ meet the conditions above, $\psi(w)=1$, where $w$ is the Denjoy-Wolff point of $\varphi$. Several examples of hyperbolic weighted composition operators on $H\left(B_{N}\right)$ are shown in Corollary 4.6 and Corollary 4.7. In the final part of this paper, the adjoint of weighted composition operator will be studied, and some special conditions will be given for such an operator to be hypercyclic.

\section{Preliminary results}

First of all, an analogue of the Denjoy-Wolff Theorem in one complex variable is presented as the following (MacCluer [18]):

Theorem 2.1 (Denjoy-Wolff Theorem in $B_{N}$ ). If $\varphi$ is a holomorphic selfmap of the open unit ball $B_{N}$ without interior fixed point, there will be a point $w \in \partial B_{N}$ so that the iterate $\left\{\varphi_{n}\right\}$ of $\varphi$ converges to $w$ uniformly on compact subsets of $B_{N}$.

The boundary point $w$ will be called the Denjoy-Wolff point of $\varphi$. In addition, the following inequality can be derived from Theorem 1.3 of [18]:

$$
0<\liminf _{z \rightarrow w} \frac{1-|\varphi(z)|^{2}}{1-|z|^{2}}=\delta \leq 1
$$

The real number $\delta$ is referred to as the boundary dilation coefficient of $\varphi$. The following is a special case of Julia's Theorem on the ball (cf. [18] and [20]): 
Theorem 2.2. Let $\varphi$ be an analytic map of the unit ball into itself with DenjoyWolff point $w \in \partial B_{N}$ and boundary dilatation coefficient $\delta$. Then

$$
\frac{|1-\langle\varphi(z), w\rangle|^{2}}{1-|\varphi(z)|^{2}} \leq \delta \frac{|1-\langle z, w\rangle|^{2}}{1-|z|^{2}}
$$

for every $z \in B_{N}$.

Definition 2.3. A holomorphic self-map $\varphi$ of $B_{N}$ will be called elliptic if $\varphi$ fixes some point of $B_{N}$; parabolic if $\varphi$ has no interior fixed point and boundary dilatation coefficient $\delta=1$; and hyperbolic if $\varphi$ has no interior fixed point and boundary dilatation coefficient $\delta<1$.

In [10], Cowen and MacCluer defined and studied a class of linear fractional maps of $B_{N}$ into itself which generalize the automorphism of $B_{N}$.

Definition 2.4. A map $\varphi$ will be called a linear fractional map if

$$
\varphi(z)=(A z+B)(\langle z, C\rangle+D)^{-1},
$$

where $A$ is an $N \times N$ matrix, $B$ and $C$ are (column) vectors in $\mathbb{C}^{N}$, and $D$ is a complex number.

If $\varphi\left(B_{N}\right) \subset B_{N}, \varphi$ is said to be a linear fractional self-map of $B_{N}$ and signed as $\varphi \in \operatorname{LFM}\left(B_{N}, B_{N}\right)$. In this case, $\varphi$ is actually holomorphic on $B_{N}$ only if the inequality

$$
|D| \geq|C|
$$

holds.

Definition 2.5. A slice $S$ of $B_{N}$ is a non-empty subset of $B_{N}$ of the form $S=B_{N} \bigcap V$, where $V$ is an one dimensional affine subspace of $\mathbb{C}^{N}$. We say that a slice $S$ of $B_{N}$ passes through some point $z \in B_{N}$ if $z \in \bar{S}$. Likewise, a vector $v \in \mathbb{C}^{N} \backslash\{0\}$ is a direction vector of $S$ if

$$
v \in V_{S}:=\operatorname{span}\left\{s-s^{\prime}: s, s^{\prime} \in S\right\} .
$$

This one-dimensional vector space $V_{S}$ is termed the direction subspace of $S$.

A finite collection $\left\{S_{1}, \ldots, S_{p}\right\}$ of slices of $B_{N}$ is said to be independent if the family of the corresponding one-dimensional direction subspaces $\left\{V_{S_{1}}, \ldots, V_{S_{p}}\right\}$ spans a $p$-dimensional subspace of $\mathbb{C}^{N}$. A slice $S$ is invariant (as a set) for $\varphi \in \operatorname{LFM}\left(B_{N}, B_{N}\right)$ if $\varphi(S) \subset S$.

Let $\varphi \in \operatorname{LFM}\left(B_{N}, B_{N}\right), \# \operatorname{inv}(\varphi)$ correspond to the dimension of the space spanned by the direction subspaces $V_{S}$ of all invariant slices $S \subset B_{N}$ of $\varphi$. Obviously, \#inv $(\varphi)=0$ if and only if $\varphi$ has no invariant slice; \#inv $(\varphi)=1$ if and only if $\varphi$ has only one invariant slice (Further results about linear fractional maps and invariant slices could be found in [5]). 


\section{Properties of linear fractional self-map of $B_{N}$}

Theorem 3.1. Let $\varphi$ be a hyperbolic self-map of $B_{N}$ and $\alpha>0$. Then $\sum_{n=1}^{\infty}\left(1-\left|\varphi_{n}(z)\right|\right)^{\alpha}$ converges uniformly on compact subsets of $B_{N}$.

Proof. Let $w$ be the Denjoy-Wolff fixed point of $\varphi$ and $\delta \in(0,1)$ be the corresponding boundary dilation coefficient. Resulting from Theorem 2.2,

$$
\frac{|1-\langle\varphi(z), w\rangle|^{2}}{1-|\varphi(z)|^{2}} \leq \delta \frac{|1-\langle z, w\rangle|^{2}}{1-|z|^{2}}
$$

for all $z \in B_{N}$. As a consequence,

$$
\frac{\left|1-\left\langle\varphi_{n}(z), w\right\rangle\right|^{2}}{1-\left|\varphi_{n}(z)\right|^{2}} \leq \delta^{2} \frac{\left|1-\left\langle\varphi_{n-1}(z), w\right\rangle\right|^{2}}{1-\left|\varphi_{n-1}(z)\right|^{2}} \leq \cdots \leq \delta^{n} \frac{|1-\langle z, w\rangle|^{2}}{1-|z|^{2}}
$$

for every $z \in B_{N}$ and every nonnegative integer $n$. If $K$ is a compact subset of $B_{N}$, there is a constant $M>0$ such that $\frac{|1-\langle z, w\rangle|^{2}}{1-|z|^{2}}<M$ for all $z$ in $K$. Therefore,

$$
\begin{aligned}
1-\left|\varphi_{n}(z)\right|^{2} & \leq 2\left(1-\left|\varphi_{n}(z)\right|\right) \leq 2\left|1-\left\langle\varphi_{n}(z), w\right\rangle\right| \\
& \leq 2 \frac{\left|1-\left\langle\varphi_{n}(z), w\right\rangle\right|^{2}}{\left|1-\left\langle\varphi_{n}(z), w\right\rangle\right|} \leq 4 \frac{\left|1-\left\langle\varphi_{n}(z), w\right\rangle\right|^{2}}{1-\left|\varphi_{n}(z)\right|^{2}} \\
& \leq 4 \delta^{n} \frac{|1-\langle z, w\rangle|^{2}}{1-|z|^{2}} \leq 4 M \delta^{n}
\end{aligned}
$$

and consequently $\left(1-\left|\varphi_{n}(z)\right|\right)^{\alpha} \leq(4 M)^{\alpha} \delta^{\alpha n}$. Thus $\sum_{i=1}^{\infty}\left(1-\left|\varphi_{i}(z)\right|\right)^{\alpha}$ converges uniformly on compact subsets of $B_{N}$.

Theorem 3.2. Let $\varphi$ be a parabolic linear fractional self-map of $B_{N}$ with at least one invariant slice. If the restriction of $\varphi$ to one of its invariant slices is an automorphism, then $\sum_{n=1}^{\infty}\left(1-\left|\varphi_{n}(z)\right|\right)^{\alpha}$ converges uniformly on compact subsets of $B_{N}$ if and only if $\alpha>1 / 2$. Otherwise, $\sum_{n=1}^{\infty}\left(1-\left|\varphi_{n}(z)\right|\right)^{\alpha}$ converges uniformly on compact subsets if and only if $\alpha>1$.

Proof. Without loss of generality, we may conjugate $\varphi$ by a rotation of $\mathbb{C}^{N}$ and assume that the Denjoy-Wolff point $w=(1,0, \ldots, 0)$. It will be convenient to move the problem to the Siegel right half-space:

$$
\mathbb{H}^{N}=\left\{\left(u_{1}, u^{\prime}\right) \in \mathbb{C} \times \mathbb{C}^{N-1}: \operatorname{Re} u_{1} \geq\left|u^{\prime}\right|^{2}\right\}
$$

which is biholomorphically equivalent to $B_{N}$ via the generalized Cayley transform

$$
\sigma\left(z_{1}, z^{\prime}\right)=\left(\frac{1+z_{1}}{1-z_{1}}, \frac{z^{\prime}}{1-z_{1}}\right) .
$$

The inverse of $\sigma$ is given by

$$
\sigma^{-1}\left(u_{1}, u^{\prime}\right)=\left(\frac{u_{1}-1}{1+u_{1}}, \frac{2 u^{\prime}}{1+u_{1}}\right) .
$$


Easy calculations show that for any $z=\left(z_{1}, z^{\prime}\right) \in B_{N}$ and $\left(u_{1}, u^{\prime}\right)=\sigma(z)$,

$$
1-|z|^{2}=\frac{4}{\left|u_{1}+1\right|^{2}}\left(\operatorname{Re} u_{1}-\left|u^{\prime}\right|^{2}\right) .
$$

Owing to Lemma 4.1 in [5], $\varphi$ is conjugate to a map $\tilde{\varphi}: \mathbb{H}^{N} \rightarrow \mathbb{H}^{N}$ of the form

$$
\tilde{\varphi}\left(u_{1}, u^{\prime}\right)=\left(u_{1}+c+\left\langle u^{\prime}, b\right\rangle, A u^{\prime}+d\right)
$$

for suitable scalar $c \in \mathbb{C}$, vectors $b, d \in \mathbb{C}^{N-1}$ and $(N-1) \times(N-1)$ matrix $A$ with $\|A\| \leq 1$ where $\|A\|$ is the spectrum norm of $A$. Of course these parameters satisfy a number of relations, determined by the condition that $\tilde{\varphi}$ maps $\mathbb{H}^{N}$ into itself.

A self-map $\eta$ of $\mathbb{H}^{N}$ defined by

$$
\eta\left(u_{1}, u^{\prime}\right)=\left(u_{1}+2\left\langle u^{\prime}, k_{2}\right\rangle+k_{1}, u^{\prime}+k_{2}\right),\left(u_{1}, u^{\prime}\right) \in \mathbb{H}^{N}
$$

with $\left(k_{1}, k_{2}\right) \in \partial \mathbb{H}^{N}$ is said to be a Heisenberg translation. According to Proposition 4.3 of $[5], \eta \in \operatorname{Aut}\left(\mathbb{H}^{N}\right)$. Let

$$
\tilde{\tilde{\varphi}}=\eta^{-1} \circ \tilde{\varphi} \circ \eta \text {. }
$$

Straightforward computations show that

$$
\begin{aligned}
\tilde{\tilde{\varphi}}\left(u_{1}, u^{\prime}\right) & =\left(u_{1}+\left\langle u^{\prime}, b+2 k_{2}-2 A^{*} k_{2}\right\rangle+\tilde{c}, A u^{\prime}+A k_{2}+d-k_{2}\right) \\
& =\left(u_{1}+\left\langle u^{\prime}, \tilde{b}\right\rangle+\tilde{c}, A u^{\prime}+\tilde{d}\right)
\end{aligned}
$$

for some $\tilde{c} \in \mathbb{C}$ and $\tilde{b}=b+2 k_{2}-2 A^{*} k_{2}, \tilde{d}=A k_{2}+d-k_{2}$.

If $\varphi \in \operatorname{LFM}\left(B_{N}, B_{N}\right)$ and $\# \operatorname{inv}(\varphi) \geq 1$, by the proof of Proposition 4.4 in [5], there is $u_{0}^{\prime} \in \mathbb{C}^{N-1}$ such that $\tilde{\varphi}\left(\left\{\left.\left(u_{1}, u_{0}^{\prime}\right)\left|\operatorname{Re}\left(u_{1}\right) \geq\right| u_{0}^{\prime}\right|^{2}\right\}\right) \subset\left\{\left(u_{1}, u_{0}^{\prime}\right) \mid \operatorname{Re}\left(u_{1}\right)\right.$ $\left.\geq\left|u_{0}^{\prime}\right|^{2}\right\}$. Therefore $A u_{0}^{\prime}+d=u_{0}^{\prime}$. Let $k_{2}=u_{0}^{\prime}$ and $k_{1}=\left|k_{2}\right|^{2}$, then

$$
\tilde{\tilde{\varphi}}\left(u_{1}, u^{\prime}\right)=\left(u_{1}+\left\langle u^{\prime}, \tilde{b}\right\rangle+\tilde{c}, A u^{\prime}\right)
$$

for some $\tilde{b} \in \mathbb{C}^{N-1}$ and $\tilde{c} \in \mathbb{C}$. If $\tilde{c}=0$, then $\tilde{\tilde{\varphi}}$ fixes points of the form $\left(u_{1}, O^{\prime}\right)$ for all $u_{1}$ with $\operatorname{Re} u_{1}>0$, which implies that $\varphi$ has an interior fixed point and this is impossible since $\varphi$ is non-elliptic. Therefore $\tilde{c} \neq 0$ and

$$
\tilde{\tilde{\varphi}}_{n}\left(u_{1}, u^{\prime}\right)=\left(u_{1}+\left\langle u^{\prime}, \sum_{k=0}^{n-1}\left(A^{*}\right)^{k} \tilde{b}\right\rangle+n \tilde{c}, A^{n} u^{\prime}\right) .
$$

If $\varphi \in \operatorname{LFM}\left(B_{N}, B_{N}\right)$ and has only one invariant slice, that is $\# \operatorname{inv}(\varphi)=1$, then based on Proposition 4.4 of [5], 1 is not an eigenvalue of $A$ and $E_{N-1}-A^{*}$ is invertible. Due to the following expression

$$
\sum_{k=0}^{n-1}\left(A^{*}\right)^{k}=\left(E_{N-1}-A^{*}\right)^{-1}\left(E_{N-1}-\left(A^{*}\right)^{n}\right),
$$

we obtain

$$
\begin{aligned}
\left|\left\langle u^{\prime}, \sum_{k=0}^{n-1}\left(A^{*}\right)^{k} \tilde{b}\right\rangle\right| & \leq\left|u^{\prime}\right|\left\|\left(E_{N-1}-A^{*}\right)^{-1}\left(E_{N-1}-\left(A^{*}\right)^{n}\right)\right\||\tilde{b}| \\
& \leq 2\left|u^{\prime}\right|\left\|\left(E_{N-1}-A^{*}\right)^{-1}\right\||\tilde{b}| .
\end{aligned}
$$


According to $\varphi=\sigma^{-1} \circ \eta \circ \tilde{\tilde{\varphi}} \circ \eta^{-1} \circ \sigma$ and (3.1), for every $\left(z_{1}, z^{\prime}\right) \in B_{N}$, and $\left(u_{1}, u^{\prime}\right)=\eta^{-1}\left(\sigma\left(z_{1}, z^{\prime}\right)\right)$,

$$
\begin{aligned}
& 1-\left|\varphi_{n}\left(z_{1}, z^{\prime}\right)\right|^{2} \\
= & 1-\left|\sigma^{-1} \circ \eta \circ \tilde{\tilde{\varphi}}_{n} \circ \eta^{-1} \circ \sigma\left(z_{1}, z^{\prime}\right)\right|^{2} \\
= & 1-\left|\sigma^{-1} \circ \eta \circ \tilde{\tilde{\varphi}}_{n}\left(u_{1}, u^{\prime}\right)\right|^{2} \\
= & 1-\left|\sigma^{-1}\left(u_{1}+\left\langle u^{\prime}, \sum_{k=0}^{n-1}\left(A^{*}\right)^{k} \tilde{b}\right\rangle+n \tilde{c}+2\left\langle A^{n} u^{\prime}, k_{2}\right\rangle+k_{1}, A^{n} u^{\prime}+k_{2}\right)\right|^{2} \\
= & \frac{4\left(\operatorname{Re}\left(u_{1}+\left\langle u^{\prime}, \sum_{k=0}^{n-1}\left(A^{*}\right)^{k} \tilde{b}\right\rangle+n \tilde{c}+2\left\langle A^{n} u^{\prime}, k_{2}\right\rangle+k_{1}\right)-\left|A^{n} u^{\prime}+k_{2}\right|^{2}\right)}{\left|u_{1}+\left\langle u^{\prime}, \sum_{k=0}^{n-1}\left(A^{*}\right)^{k} \tilde{b}\right\rangle+n \tilde{c}+2\left\langle A^{n} u^{\prime}, k_{2}\right\rangle+k_{1}\right|^{2}} .
\end{aligned}
$$

Due to Proposition 4.3 of [5], the restriction of $\varphi$ on its unique invariant slice is an automorphism if and only if $\operatorname{Re} \tilde{c}=0$. Consequently, if the restriction of $\varphi$ on its invariant slice is not an automorphism, and $K$ is any compact subset of $B_{N}$, for sufficiently large $n$, there exist some positive constants $M$ and $M^{\prime}$ such that

$$
\frac{M^{\prime}}{n^{\alpha}}<\left(1-\left|\varphi_{n}\left(z_{1}, z^{\prime}\right)\right|^{2}\right)^{\alpha}<\frac{M}{n^{\alpha}}(z \in K) .
$$

Because of this, $\sum_{n=1}^{\infty}\left(1-\left|\varphi_{n}\left(z_{1}, z^{\prime}\right)\right|^{2}\right)^{\alpha}$ converges uniformly on compact subsets of $B_{N}$ if and only if $\alpha>1$.

On the other hand, suppose that the restriction of $\varphi$ on its unique invariant slice is an automorphism, since $\tilde{c} \neq 0$, for fixed compact subset $K$ of $B_{N}$ and sufficiently large $n$, there exist positive constants $M$ and $M^{\prime}$ such that for every $z \in K$

$$
\frac{M^{\prime}}{n^{2 \alpha}}<\left(1-\left|\varphi_{n}\left(z_{1}, z^{\prime}\right)\right|^{2}\right)^{\alpha}<\frac{M}{n^{2 \alpha}} .
$$

Hence $\sum_{n=1}^{\infty}\left(1-\left|\varphi_{n}\left(z_{1}, z^{\prime}\right)\right|^{2}\right)^{\alpha}$ converges uniformly on compact subsets of $B_{N}$ if and only if $\alpha>\frac{1}{2}$.

If $\varphi \in \operatorname{LFM}\left(B_{N}, B_{N}\right)$ and $q+1:=\# \operatorname{inv}(\varphi)>1$, resulting from $(3.3), \varphi$ is conjugate to $\tilde{\tilde{\varphi}}$ where

$$
\tilde{\tilde{\varphi}}\left(u_{1}, u^{\prime}\right)=\left(u_{1}+\left\langle u^{\prime}, \tilde{b}\right\rangle+\tilde{c}, A u^{\prime}\right) .
$$

There is a unitary matrix $U$ such that (cf. Lemma 4.4 in [5])

$$
\tilde{A}:=U^{*} A U=\left[\begin{array}{ll}
E_{q} & O \\
O & B
\end{array}\right],
$$

where $B \in \mathbb{C}^{N-1-q} \times \mathbb{C}^{N-1-q}$ with $|B| \leq 1$ and 1 is not a eigenvalue of $B$. Let $\tilde{U}\left(u_{1}, u^{\prime}\right)=\left(u_{1}, U u^{\prime}\right)$ and

$$
\begin{aligned}
\varphi^{1}\left(u_{1}, u^{\prime}\right) & =\tilde{U}^{-1} \circ \tilde{\tilde{\varphi}} \circ \tilde{U}\left(u_{1}, u^{\prime}\right) \\
& =\left(u_{1}+\left\langle u^{\prime *} \tilde{b}\right\rangle+\tilde{c}, \tilde{A} u^{\prime}\right) .
\end{aligned}
$$


Write $U^{*} \tilde{b}=\left(\tilde{b}^{\prime \prime}, \tilde{b}^{\prime \prime \prime}\right) \in \mathbb{C}^{q} \times \mathbb{C}^{N-1-q}$ then

$$
\varphi^{1}\left(u_{1}, u^{\prime}\right)=\left(u_{1}+\left\langle u^{\prime \prime}, \tilde{b}^{\prime \prime}\right\rangle+\left\langle u^{\prime \prime \prime}, \tilde{b}^{\prime \prime \prime}\right\rangle+c, u^{\prime \prime}, B u^{\prime \prime \prime}\right)
$$

with $\left(u_{1}, u^{\prime}\right)=\left(u_{1}, u^{\prime \prime}, u^{\prime \prime \prime}\right) \in \mathbb{C} \times \mathbb{C}^{q} \times \mathbb{C}^{N-1-q} \cap \mathbb{H}^{N}$. According to Proposition 4.2 of [5], since $\varphi^{1}$ is a self map of $\mathbb{H}^{N}, \tilde{b}$ belongs to the space spanned by columns of $E_{N-1}-\tilde{A}^{*} \tilde{A}$. Hence $\tilde{b}^{\prime \prime}=0$ and

$$
\varphi_{n}^{1}\left(u_{1}, u^{\prime \prime}, u^{\prime \prime \prime}\right)=\left(u_{1}+\left\langle u^{\prime \prime \prime}, \sum_{k=0}^{n-1}\left(B^{*}\right)^{n} \tilde{b}^{\prime \prime \prime}\right\rangle+n \tilde{c}, u^{\prime \prime}, B^{n} u^{\prime \prime \prime}\right) .
$$

The rest discussion of the present lemma follows by the same discussion as the case of $\# \operatorname{inv}(\varphi)=1$.

Theorem 3.3. Let $\varphi$ be a parabolic automorphism with $\# \operatorname{inv}(\varphi)=0$. Then $\sum_{n=1}^{\infty}\left(1-\left|\varphi_{n}(z)\right|\right)^{\alpha}$ converges uniformly on compact subsets of $B_{N}$ if and only if $\alpha>\frac{1}{4}$.

Proof. Without loss of generality, we may assume that the Denjoy-Wolff point $w=(1,0, \ldots, 0)$. According to $(3.2), \varphi$ is conjugated to

$$
\tilde{\varphi}\left(u_{1}, u^{\prime}\right)=\left(u_{1}+\left\langle u^{\prime}, b\right\rangle+c, A u^{\prime}+d\right) .
$$

Since $\tilde{\varphi}$ is an automorphism of $\mathbb{H}^{N}, A$ is a unitary matrix and $b=2 A^{*} d$. Owing to Proposition 4.4 of [5], 1 is an eigenvalue of $A$. Moreover, resulting from the spectral theorem, there is a unitary matrix $V$ of order $n-1$ such that $V^{*} A V=\Theta$, where

$$
\Theta=\left[\begin{array}{cc}
E_{q} & O \\
O & \Lambda
\end{array}\right]
$$

with $E_{q}$ the identity matrix of order $q$ and $\Lambda$ a diagonal unitary matrix of order $N-q-1$ with diagonal elements of the form $e^{i t}(t \neq 0)$.

Set $\tilde{V}\left(u_{1}, u^{\prime}\right):=\left(u_{1}, V u^{\prime}\right)$ and

$$
\begin{aligned}
\varphi^{2}\left(u_{1}, u^{\prime}\right) & :=\tilde{V}^{-1} \circ \tilde{\varphi} \circ \tilde{V}\left(u_{1}, u^{\prime}\right) \\
& =\left(u_{1}+\left\langle u^{\prime}, V^{*} b\right\rangle+c, \Theta u^{\prime}+V^{*} d\right) .
\end{aligned}
$$

Let $V^{*} d=\left(d^{\prime \prime}, d^{\prime \prime \prime}\right) \in \mathbb{C}^{q} \times \mathbb{C}^{N-1-q}$, then

$$
V^{*} b=2 V^{*} A^{*} V V^{*} d=2\left[\begin{array}{ll}
E_{q} & O \\
O & \Lambda^{*}
\end{array}\right] V^{*} d=\left(2 d^{\prime \prime}, 2 \Lambda^{*} d^{\prime \prime \prime}\right) .
$$

Therefore

$$
\varphi^{2}\left(u_{1}, u^{\prime}\right)=\left(u_{1}+2\left\langle u^{\prime \prime}, d^{\prime \prime}\right\rangle+2\left\langle u^{\prime \prime \prime}, \Lambda^{*} d^{\prime \prime \prime}\right\rangle+c, u^{\prime \prime}+d^{\prime \prime}, \Lambda u^{\prime \prime \prime}+d^{\prime \prime \prime}\right)
$$

with $\left(u_{1}, u^{\prime}\right)=\left(u_{1}, u^{\prime \prime}, u^{\prime \prime \prime}\right) \in \mathbb{C} \times \mathbb{C}^{q} \times \mathbb{C}^{N-1-q} \cap \mathbb{H}^{N}$. Owing to conjugating with the Heisenberg translation

$$
\eta_{1}\left(u_{1}, u^{\prime \prime}, u^{\prime \prime \prime}\right)=\left(u_{1}+2\left\langle u^{\prime \prime \prime}, k_{2}\right\rangle+k_{1}, w^{\prime \prime}, w^{\prime \prime \prime}+k_{2}\right),
$$


where $\left(k_{1}, O, k_{2}\right) \in \partial \mathbb{H}^{N}$ and $k_{2}=\left(E_{m-1-q}-\Lambda\right)^{-1} d^{\prime \prime \prime}$, we obtain a new automorphism

$$
\varphi^{3}\left(u_{1}, u^{\prime \prime}, u^{\prime \prime \prime}\right)=\left(z+2\left\langle u^{\prime \prime}, d^{\prime \prime}\right\rangle+\tilde{c}, u^{\prime \prime}+d^{\prime \prime}, \Lambda u^{\prime \prime \prime}\right)
$$

for some $\tilde{c} \in \mathbb{C}$ with $\operatorname{Re}(\tilde{c})=\left|d^{\prime \prime}\right|^{2}$. With respect to Proposition 4.4 of [5], $\varphi^{3}$ has no invariant slice if and only if $d^{\prime \prime} \neq O$. Direct computations obtain that

$$
\varphi_{n}^{3}\left(u_{1}, u^{\prime \prime}, u^{\prime \prime \prime}\right)=\left(z+2 n\left\langle u^{\prime \prime}, d^{\prime \prime}\right\rangle+\left(n^{2}-n\right)\left|d^{\prime \prime}\right|^{2}+n \tilde{c}, u^{\prime \prime}+n d^{\prime \prime}, \Lambda^{n} u^{\prime \prime \prime}\right) .
$$

Let $\left(u_{1}, u^{\prime \prime}, u^{\prime \prime \prime}\right)=\eta_{1}^{-1} \circ \tilde{V}^{-1} \circ \sigma\left(z_{1}, z^{\prime}\right)$ for $\left(z_{1}, z^{\prime}\right) \in B_{N}$, notice that

$$
\varphi=\sigma^{-1} \circ \tilde{V} \circ \eta_{1} \circ \varphi^{3} \circ \eta_{1}^{-1} \circ \tilde{V}^{-1} \circ \sigma,
$$

we get

$$
\begin{aligned}
& 1-\left|\varphi_{n}\left(z_{1}, z^{\prime}\right)\right|^{2}=1-\left|\sigma^{-1} \circ \tilde{V} \circ \eta_{1} \circ \varphi_{n}^{3}\left(u_{1}, u^{\prime \prime}, u^{\prime \prime \prime}\right)\right|^{2} \\
= & 1-\mid \sigma^{-1} \circ \tilde{V} \circ \eta_{1}\left(z+2 n\left\langle u^{\prime \prime}, d^{\prime \prime}\right\rangle+\left(n^{2}-n\right)\left|d^{\prime \prime}\right|^{2}\right. \\
& \left.+n \tilde{c}, u^{\prime \prime}+n d^{\prime \prime}, \Lambda^{n} u^{\prime \prime \prime}\right)\left.\right|^{2} \\
= & \frac{4\left(\Re\left(u_{1}+2\left\langle\Lambda^{n} u^{\prime \prime \prime}, k_{2}\right\rangle+k_{1}\right)-\left|u^{\prime \prime}\right|^{2}-\left|\Lambda^{n} w^{\prime \prime \prime}+k_{2}\right|^{2}\right)}{\left.\left|1+u_{1}+2 n\left\langle u^{\prime \prime}, d^{\prime \prime}\right\rangle+\left(n^{2}-n\right)\right| d^{\prime \prime}\right|^{2}+n \tilde{c}+2\left\langle\Lambda^{n} u^{\prime \prime \prime}, k_{2}\right\rangle+\left.k_{1}\right|^{2}} .
\end{aligned}
$$

If $K$ is any compact subset of $B_{N}$, for sufficiently large $n$, there exist some positive constants $M$ and $M^{\prime}$ such that

$$
\frac{M^{\prime}}{n^{4 \alpha}} \leq\left(1-\left|\varphi_{n}\left(z_{1}, z^{\prime}\right)\right|^{2}\right)^{\alpha} \leq \frac{M}{n^{4 \alpha}}
$$

and consequently $\sum_{n=1}^{\infty}\left(1-\left|\varphi_{n}\left(z_{1}, z^{\prime}\right)\right|^{2}\right)^{\alpha}$ converges uniformly on compact subsets of $B_{N}$ if and only if $\alpha>\frac{1}{4}$.

Proposition 3.4. Let $\varphi$ be a linear fractional self-map of the open unit ball $B_{N}$ with unique fixed point $w$ in $B_{N}$. Then $\varphi_{n}$ converges to $w$ on every compact subset of $B_{N}$ if and only if $\rho\left(d \varphi_{w}\right)<1$, where $d \varphi_{w}$ is the Jacobian matrix of $\varphi$ at $w$.

Proof. Since the spectral radius of a matrix is invariant under conjugations, we may assume that $w=O$. In this case, $\varphi$ can be given by

$$
\varphi(z)=\frac{A z}{\langle z, C\rangle+1} .
$$

Direct calculations show that $d \varphi_{O}=A$ and

$$
\varphi_{n}(z)=\frac{A^{n}(z)}{\left\langle\left(\sum_{i=0}^{n-1} A^{i}\right) z, C\right\rangle+1} .
$$

Let $\rho(A)<1$. Then $A-E$ and consequently $A^{H}-E$ are invertible. Therefore, there is a unique vector in $\mathbb{C}^{N}$ such that $C=\left(A^{H}-E\right) V$. There also 
exists a unitary matrix $U \in \mathbb{C}^{N \times N}$ such that $U^{H} V=\delta e_{1}$, where $\delta=|V|$ and $e_{1}=(1,0, \ldots, 0)^{T}$. Let $\hat{\varphi}(z)=U^{H} \circ \varphi \circ U(z)$ and $\hat{A}=U^{H} A U$, then

$$
\begin{aligned}
\hat{\varphi}(z) & =\frac{U^{H} A U z}{\langle U z, C\rangle+1}=\frac{U^{H} A U z}{\left\langle U z,\left(A^{H}-E\right) V\right\rangle+1} \\
& =\frac{U^{H} A U z}{\left\langle z, U^{H}\left(A^{H}-E\right) V\right\rangle+1}=\frac{U^{H} A U z}{\left\langle z,\left(U^{H} A^{H} U-E\right) U^{H} V\right\rangle+1} \\
& =\frac{\hat{A} z}{\delta\left\langle z,\left(\hat{A}^{H}-E\right) e_{1}\right\rangle+1} .
\end{aligned}
$$

As a consequence,

$$
\hat{\varphi}_{n}(z)=\frac{\hat{A}^{n} z}{\delta\left\langle z,\left(\left(\hat{A}^{n}\right)^{H}-E\right) e_{1}\right\rangle+1} .
$$

Because $\hat{\varphi}_{n}$ is a linear fractional self-map of $B_{N}$ for every $n \in \mathbb{N}$,

$$
\left|\delta\left(\left(\hat{A}^{n}\right)^{H}-E\right) e_{1}\right| \leq 1
$$

Since $\rho(A)=\rho(\hat{A})$, letting $n$ goes to infinity, we get $\delta \in[0,1]$. Let

$$
\sigma(z):=\frac{z}{-\delta z_{1}+1}, z=\left(z_{1}, z^{\prime}\right) \in \mathbb{C} \times \mathbb{C}^{N-1} \cap B_{N} .
$$

The linear fractional map $\sigma$ is clearly holomorphic and injective on $B_{N}$, since $\delta \leq 1$. Direct computations show that

$$
\sigma \circ \hat{\varphi}(z)=\hat{A} \sigma(z)
$$

for all $z \in B_{N}$. As a result, $\hat{\varphi}_{n}$ and consequently $\varphi_{n}$ converge uniformly on compact subsets of $B_{N}$.

On the other hand, let $\varphi_{n}$ converge to $O$ on every compact subset of $B_{N}$. Write

$$
\varphi_{n}=\left(\varphi_{n}^{1}, \varphi_{n}^{2}, \ldots, \varphi_{n}^{N}\right)
$$

and

$$
A^{n}=\left(a_{i j}^{n}\right) .
$$

Then

and by Cauchy inequality,

$$
a_{i j}^{n}=\frac{\partial \varphi_{n}^{i}}{\partial z_{j}}(O),
$$

$$
\left|a_{i j}^{n}\right| \leq 2 \sup _{|z|=\frac{1}{2}}\left|\varphi_{n}^{i}\right| .
$$

Therefore

$$
\lim _{n \rightarrow \infty}\left|a_{i j}^{n}\right|=0 .
$$


As a result,

which means that

$$
\lim _{n \rightarrow \infty} A^{n}=O
$$

$$
\rho(A)<1 \text {. }
$$

\section{Weighted composition operators on $H\left(B_{N}\right)$}

The next theorem presents a necessary condition for when a weighted composition operator is hypercyclic.

Theorem 4.1. Let $\varphi$ be a self-map of $B_{N}$ without interior fixed points. Let $w$ be the Denjoy-Wolff point of $\varphi$. Let $\psi \in \operatorname{Lip}_{2 \alpha}(w)$ for some real number $\alpha$, and $\psi$ never vanishes on $B_{N}$ and $|\psi(w)|=1$. If the composition operator $C_{\varphi}$ is hypercyclic on $H\left(B_{N}\right)$, then $W_{\psi, \varphi}$ is hypercyclic whenever $\varphi$ is a hyperbolic selfmap with $\alpha>0$ or $\varphi$ is a parabolic linear fractional self-map with at least one invariant slice and $\alpha>\frac{1}{2}$ or $\varphi$ is a parabolic automorphism without invariant slice and $\alpha>\frac{1}{4}$.

The following lemmas and propositions are needed to prove the above theorem. Most of them are direct extensions of results in [22].

Theorem 4.2. If $W_{\psi, \varphi}$ is hyperbolic on $H\left(B_{N}\right)$, then

(i) $\varphi$ has no fixed point in $B_{N}$ and $\psi(z) \neq 0$ for every $z \in B_{N}$;

(ii) $\varphi$ is univalent.

The proof is omitted. The readers are referred to Proposition 2.1 in [22] for details.

Proposition 2.3 of [22] showed that the unimodular scalar multiple of a hypercyclic composition operator is also hypercyclic. According to the following lemma, this is true in the present case.

Lemma 4.3. Let $X$ be the set of polynomials vanishing at the boundary point $w$. Then $X$ is a dense subset of $H\left(B_{N}\right)$.

Proof. It is well known that the set of polynomials vanishing in the same boundary point is dense in $H^{2}(U)$, which is the Hardy space of the unit disc of $\mathbb{C}$. The same result will be shown to hold in $H^{2}\left(B_{N}\right)$.

Without loss of generality, let $w=(1,0, \ldots, 0)$. Let $Y$ denote the set of polynomials of one variable vanishing at 1 . Therefore $Y$ is a dense subset of $H^{2}(U)$. Since $f \equiv 1$ belongs to $H^{2}(U)$, there is a sequence $\left\{f_{k}, k=1,2, \ldots\right\}$ of $Y$ tending to $f$ in $H^{2}(U)$. For every polynomial $g \in H^{2}\left(B_{N}\right)$ and every $z=\left(z_{1}, z^{\prime}\right) \in \mathbb{C} \times \mathbb{C}^{n-1}$, let $g_{k}(z)=g(z) \cdot f_{k}\left(z_{1}\right)(k=1,2, \ldots)$. It is easy to see that $g_{k} \in X$ for every $k$. And

$$
\left\|g-g_{k}\right\|_{H^{2}\left(B_{N}\right)}=\left\|g\left(1-f_{k}\right)\right\|_{H^{2}\left(B_{N}\right)} \leq C\|g\|_{H^{2}\left(B_{N}\right)}\left\|1-f_{k}\right\|_{H^{2}(U)}
$$

for some non-zero constant $C$. As a result, $X$ is a dense subset of the set of polynomials in $H^{2}\left(B_{N}\right)$. Due to Corollary 4.26 of [24], the set of polynomials is dense in $H^{2}\left(B_{N}\right)$, we conclude that $X$ is dense in $H^{2}\left(B_{N}\right)$. 
Since convergence in the Hardy space $H^{2}\left(B_{N}\right)$ implies convergence in $H\left(B_{N}\right)$ (cf. Theorem 4.17 in [24]), with both spaces containing the polynomials as a dense subset, the proof is completed.

Proposition 4.4. Let $C_{\varphi}$ be hypercyclic on $H\left(B_{N}\right)$. Then $\lambda C_{\varphi}$ is hypercyclic for every complex number $\lambda$ with $|\lambda|=1$.

Proof. Since $C_{\varphi}$ is hypercyclic, $\varphi$ has no fixed point in $B_{N}$ and there is $w \in \partial B_{N}$ such that $\varphi_{k}(z) \rightarrow w$ for every $z \in B_{N}$. Let $X$ be the set stated in the preceding lemma. For every $f \in X, C_{\varphi}^{n}(f)=f \circ \varphi_{n}$ tends to zero as $n \rightarrow \infty$. The hypercyclicity of $\lambda C_{\varphi}$ follows similarly from [22, Proposition 2.3].

Bounded operators $T$ and $S$ on an F-space $X$ are quasi-similar if there exists a bounded operator $V$ on $X$ which has dense range and $T V=V S$. If $V$ is invertible, $T$ and $S$ are said to be similar. The similarity and quasi-similarity preserve hypercyclicity. Indeed, if $x$ is a hypercyclic vector for $S, V(x)$ is a hypercyclic vector for $T$.

The existence of nonzero eigenvalues of a weighted composition operator $W_{\psi, \varphi}$ makes it possible to show that $W_{\psi, \varphi}$ inherits the hypercyclicity of $C_{\varphi}$ at least in some cases. In fact, if $\lambda$ is a nonzero eigenvalue of $W_{\psi, \varphi}$, there will be a nonzero holomorphic function $g$ with $W_{\psi, \varphi}(g)=\lambda g$. In particular, $W_{\psi, \varphi} M_{g}=M_{g}\left(\lambda C_{\varphi}\right)$. If $\lambda$ is unimodular and $g$ never vanishes in $B_{N}, M_{g}$ will be one-to-one and have dense range. As a consequence, $W_{\psi, \varphi}$ is quasi-similar to $\lambda C_{\varphi}$, which is hypercyclic by Proposition 4.4. Thus $W_{\psi, \varphi}$ is also hypercyclic.

Proposition 4.5. Let $\varphi$ be a self-map of $B_{N}$ and $w$ be the Denjoy-Wolff point of $\varphi$. Let $\psi \in \operatorname{Lip}_{2 \alpha}(w)$ for some real number $\alpha$, and $\psi(w) \neq 0 . \psi(w)$ is an eigenvalue for $W_{\psi, \varphi}$, if $\varphi$ and $\alpha$ satisfy any of the following hypotheses:

(1) $\varphi$ is a hyperbolic self-map of $B_{N}$ and $\alpha>0$;

(2) $\varphi$ is a parabolic linear fractional self-map of $B_{N}$ with \#inv $(\varphi)>0$ and the constrain of $\varphi$ to one of its invariant slice is an automorphism and $\alpha>1 / 2$

(3) $\varphi$ is a parabolic linear fractional self-map of $B_{N}$ with \#inv $(\varphi)>0$ and $\alpha>1$

(4) $\varphi$ is a parabolic automorphism with \#inv $(\varphi)=0$ and $\alpha>1 / 4$.

Proof. Our hypotheses show that there exist some constant $M$ and $\sigma>0$, such that

$$
|\psi(z)-\psi(w)| \leq M|z-w|^{2 \alpha}
$$

for every $z$ with $|z-w| \leq \sigma$. Let $K$ be a compact subset of $B_{N}$. Theorem 2.2 provides a constant $c>0$ such that

$$
\left|1-\left\langle\varphi_{n}(z), w\right\rangle\right|^{2} \leq c\left(1-\left|\varphi_{n}(z)\right|^{2}\right)
$$

and therefore

$$
\left|1-\left\langle\varphi_{n}(z), w\right\rangle\right|^{2 \alpha} \leq c^{\alpha}\left(1-\left|\varphi_{n}(z)\right|^{2}\right)^{\alpha}
$$


for every $z \in K$ and every $n \in \mathbb{N}$. Due to substituting $\varphi_{n}(z)$ instead of $z$ in inequality (4.1), and $\left|\varphi_{n}(z)-w\right|=\left|1-\left\langle\varphi_{n}(z), w\right\rangle\right|$, we obtain that

$$
\left|\psi\left(\varphi_{n}(z)\right)-\psi(w)\right| \leq M c^{\alpha}\left(1-\left|\varphi_{n}(z)\right|^{2}\right)^{\alpha}
$$

for every $n>N$. Consequently,

$$
\left|1-\frac{\psi\left(\varphi_{n}(z)\right)}{\psi(w)}\right| \leq \frac{M c^{\alpha}}{|\psi(w)|}\left(1-\left|\varphi_{n}(z)\right|^{2}\right)^{\alpha} .
$$

As a result of Theorem 3.1, Theorem 3.2 or Theorem 3.3, $\sum_{n=0}^{\infty}\left(1-\left|\varphi_{n}(z)\right|^{2}\right)^{\alpha}$ and consequently $\sum_{n=0}^{\infty}\left|1-\frac{\psi\left(\varphi_{n}(z)\right)}{\psi(w)}\right|$ converges uniformly on $K$ if $\varphi$ and $\alpha$ satisfy one of the hypetheses. Thus $\prod_{n=0}^{\infty} \frac{\psi\left(\varphi_{n}(z)\right)}{\psi(w)}$ also converges uniformly on $K$. Define

$$
g(z)=\prod_{n=0}^{\infty} \frac{\psi\left(\varphi_{n}(z)\right)}{\psi(w)} .
$$

Thus $g$ is a well defined holomorphic function in $B_{N}$. Since $\psi(w) \neq 0$, there is a neighborhood $U \in B_{N}$ of $w$ such that $\psi$ never vanishes on $B_{N}$ and so does $\psi \circ \varphi_{n}$ for every $n$ by Theorem 2.2 . For given $z_{0}^{\prime} \in \mathbb{C}^{N-1}$, let

$$
h\left(z_{1}\right)=g\left(z_{1}, z_{0}^{\prime}\right)
$$

with $\left(z_{1}, z_{0}^{\prime}\right) \in B_{N}$. Due to Theorem 5.9 of [8], $h\left(z_{1}\right)$ and consequently $g$ are nonzero holomorphic functions with respect to $z_{1}$. Same arguments show that $g$ is holomorphic with respect to other variables. Therefore, $g$ is holomorphic on $B_{N}$.

Since $W_{\psi, \varphi}(g)(z)=\psi(w) g(z), \psi(w)$ is a eigenvalue for $W_{\psi, \varphi}$ and $g$ is the corresponding eigenfunction.

It must be noted that if $\psi$ never vanishes on $B_{N}$, according to Theorem 5.9 of [8] and the discussions above, $g$ never vanishes on $B_{N}$.

Now we return the proof of Theorem 4.1 .

Proof of Theorem 4.1. Due to Proposition 4.5, $\psi(w)$ is a eigenvalue of $\varphi$. The result follows from Proposition 4.4.

Since $H^{2}\left(B_{N}\right)$ is dense in $H\left(B_{N}\right)$, a hypercyclic operator on $H^{2}\left(B_{N}\right)$ is also hypercyclic on $H\left(B_{N}\right)$.

In [7] Chen et al. proved that the composition operator $C_{\varphi}$ is hypercyclic if $\varphi$ is an automorphism of $B_{N}$ without interior fixed point. Together with Theorem 4.1, we get

Corollary 4.6. Let $\varphi$ be an automorphism of $B_{N}$ without interior fixed point and Denjoy-Wolff point $w$. Let $\psi \in$ Lip $_{2 \alpha}(w)$ for some real number $\alpha$, never vanish on $B_{N}$ and $|\psi(w)|=1$. Then $W_{\psi, \varphi}$ is hypercyclic whenever $\varphi$ is hyperbolic with $\alpha>0$ or $\varphi$ is parabolic with at least one invariant slice and $\alpha>\frac{1}{2}$ or $\varphi$ is parabolic without invariant slice and $\alpha>\frac{1}{4}$. 
Bisi and Bracci [2] pointed out that $C_{\varphi}$ is hypercyclic if and only if its differential is injective at some point when $\varphi$ is not an automorphism and has exactly two boundary fixed points. If a linear fractional map has more than 2 fixed points, then it has at least one invariant slice. Together with Theorem 4.1 again, the following corollary holds.

Corollary 4.7. Let $\varphi \in \operatorname{LFM}\left(B_{N}, B_{N}\right)$ with Denjoy-Wolff point $w$ in $\partial B_{N}$ which is not an automorphism. $\psi \in$ Lip $_{2 \alpha}(w)$ never vanishes on $B_{N}$ with $\alpha>1$ and $|\psi(w)|=1$. If $\varphi$ has exactly two boundary fixed points and its differential is injective at some point, then $W_{\psi, \varphi}$ is hypercyclic on $H\left(B_{N}\right)$.

The ideals above may be adapted to prove an eigenvalue result for $W_{\psi, \varphi}$ acting on $H\left(B_{N}\right)$.

Proposition 4.8. Let $\varphi$ be an linear fractional self-map of $B_{N}$ with a fixed point $w \in B_{N}$ and $\rho\left(d \varphi_{w}\right)<1$. If $\psi(w) \neq 0$, then $\psi(w)$ is an eigenvalue for the operator $W_{\psi, \varphi}$ acting on $H\left(B_{N}\right)$.

Proof. Without loss of generality, we may assume that $w=O$. Since $\rho\left(d \varphi_{w}\right)<$ 1 , there is a matrix norm $\|\cdot\|_{\alpha}$ on $\mathbb{C}^{N \times N}$ such that $\left\|d \varphi_{w}\right\|_{\alpha}<\delta<1$. Let $|\cdot|_{\alpha}: \mathbb{C}^{N} \rightarrow \mathbb{R}$ be a norm compatible to $\left(\mathbb{C}^{N \times N},\|\cdot\|_{\alpha}\right) .\left|d \varphi_{w}(z)\right|_{\alpha}<\delta|z|_{\alpha}$ when $z$ is sufficiently near to zero. If $K$ is a compact subset of $B_{N}$, according to Proposition 3.4, $\varphi_{n}(z) \rightarrow 0(n \rightarrow \infty)$ uniformly on $K$. As a result, $\left|\varphi_{n+k}(z)\right|_{\alpha}<\delta^{k}\left|\varphi_{n}(z)\right|_{\alpha}$ for sufficiently large $n$, every $k \in \mathbb{N}$, and every $z \in K$. This implies that $\sum_{i=0}^{\infty}\left|\varphi_{n}(z)\right|_{\alpha}$ converges uniformly on compact subsets of $B_{N}$. Since any norm defined on $\mathbb{C}^{N}$ is equivalent, $\sum_{i=1}^{\infty}\left|\varphi_{n}(z)\right|$ converges uniformly on compact subsets of $B_{N}$. Since $\psi$ is bounded, an application of Schwarz's lemma in $B_{N}$ (cf. [20]) shows that there exists a constant $M>0$ such that $|\psi(O)-\psi(z)|<M|z|$ for every $z \in B_{N}$. But $\psi(O) \neq 0$, thus

$$
\left|1-\frac{1}{\psi(O)} \psi(z)\right|<\frac{M}{|\psi(O)|}|z| \quad\left(z \in B_{N}\right) .
$$

By substituting $\varphi_{n}(z)$ instead of $z$ in the above inequality, we get that

$$
\sum_{i=1}^{\infty}\left|1-\frac{1}{\psi(O)} \psi\left(\varphi_{i}(z)\right)\right|
$$

and consequently $g(z)=\prod_{i=0}^{\infty} \frac{1}{\psi(O)} \psi\left(\varphi_{i}(z)\right)$ converges uniformly on compact subsets of $B_{N}$ and $g$ is a nonzero holomorphic function on $B_{N}$. Besides, $\psi \cdot g \circ$ $\varphi=\psi(O) g$. The rest of this proof follows from [22], we omit it here.

\section{Adjoints of weighted composition operators on Hilbert spaces of analytic functions}

Throughout this section let $H$ be a Hilbert space of holomorphic functions on the open unit ball $B_{N}$ such that for every $z \in B_{N}$, the evaluation function $e_{z}: H \rightarrow \mathbb{C}$ defined by $e_{z}(f)=f(z)$ is bounded on $H$. According to the Riesz 
Representation theorem, there is a vector $k_{z} \in H$ such that $f(z)=\left\langle f, k_{z}\right\rangle$ for every $z$ in $B_{N}$. In this section $H$ is assumed to contain the constant functions and $M(H)=H^{\infty}\left(B_{N}\right)$. Moreover, we suppose that $\varphi$ is a holomorphic selfmap of $B_{N}$ such that $C_{\varphi}$ acts boundedly on $H$, and $\psi$ is a multiplier of $H$. For each $f \in H$ and $z \in B_{N}$, we have

$$
W_{\psi, \varphi}^{*} k_{z}=C_{\varphi}^{*} M_{\psi}^{*} k_{z}=\overline{\psi(z)} k_{\varphi(z)} .
$$

The next two theorems present some conditions for when $W_{\psi, \varphi}^{*}$ is not hypercyclic. The proofs follow the idea of [22] and Propositions 4.5 and 4.8. We omit them here.

Theorem 5.1. Let $\varphi$ be a linear fractional self-map of $B_{N}$ with unique fixed point $w \in B_{N}$ and $\rho\left(d \varphi_{w}\right)<1$. If there is some $n>0$ such that $\sup _{z \in B_{N}}\left|\varphi_{n}(z)\right|$ $<1$ and $\psi(w) \neq 0$, then $\psi(w)$ is an eigenvalue for the operator $W_{\psi, \varphi}$ acting on $H$. Hence, $W_{\psi, \varphi}^{*}$ is not hypercyclic.

Theorem 5.2. Let $w$ be the Denjoy-Wolff fixed point of $\varphi, \psi \in \operatorname{Lip}_{2 \alpha}(w)$ for some real number $\alpha$, and $\psi(w) \neq 0$. In addition, let $\sup _{z \in B_{N}}|\psi(z)| \leq|\psi(w)|$. Then $\psi(w)$ is an eigenvalue for the operator $W_{\psi, \varphi}$ acting on $H$ whenever $\varphi$ is hyperbolic and $\alpha>0$ or $\varphi$ is a parabolic automorphism without invariant slice and $\alpha>\frac{1}{4}$. And as a result, $W_{\psi, \varphi}^{*}$ is not hypercyclic.

One nice condition for hypercyclicity is the Hypercyclicity Criterion which was developed independently by Kitai [17] and Gethner and Shapiro [13]. This criterion has been used to show that certain class of operators are hypercyclic. Some reformulations of this criterion were given in [23]. We use this criterion for indicating the hypercyclicity of the adjoint of $W_{\psi, \varphi}$.

Hypercyclicity Criterion. Suppose that $X$ is a separable Banach space and that $T$ is a continuous linear mapping on $X$. If there exist two dense subsets $Y$ and $Z$ in $X$ and a sequence $\left\{n_{k}\right\}$ such that:

1. $T^{n_{k}} y \rightarrow 0$ for every $y \in Y$;

2. there exist functions $S_{n_{k}}: Z \rightarrow X$ such that for every $z \in Z, S_{n_{k}} z \rightarrow 0$ and $T^{n_{k}} S_{n_{k}} z \rightarrow z$, then $T$ is hypercyclic.

Theorem 5.3. Let $\varphi$ be an automorphism with interior fixed point $w$ and $\psi \in H\left(B_{N}\right)$ satisfies

$$
|\psi(w)|<1<\lim _{|z| \rightarrow 1} \inf |\psi(z)| .
$$

Then $W_{\psi, \varphi}^{*}$ is hypercyclic.

This theorem is a direct extension of Theorem 3.3 of [22]. We will just give the outline of the proof since it follows the same way.

Proof. Let $\eta_{w}$ be the automorphism that maps $w$ to $O$ and $\eta_{w} \circ \eta_{w}=i d$. Let $\hat{\varphi}(z)=\eta_{w} \circ \varphi \circ \eta_{w}(z)$ and $\hat{\psi}=\psi \circ \eta_{w}$. Since

$$
W_{\psi, \varphi}=C_{\eta_{w}} \circ W_{\hat{\psi}, \hat{\varphi}} \circ C_{\eta_{w}}^{-1},
$$


it is enough to prove that $W_{\hat{\psi}, \hat{\varphi}}^{*}$ is hypercyclic. Since $\left|\eta^{-1}(z)\right| \rightarrow 1$ when $|z| \rightarrow 1^{-}$,

$$
\lim _{|z| \rightarrow 1^{-}} \inf |\psi(z)| \leq \lim _{|z| \rightarrow 1^{-}} \inf |\hat{\psi}(z)|
$$

Thus

$$
|\hat{\psi}(O)|<1<\lim _{|z| \rightarrow 1^{-}} \inf |\hat{\psi}(z)| .
$$

Therefore there exist some constants $\lambda_{1}, \lambda_{2}$ and positive numbers $\delta_{1}<1$ and $\delta_{2}<1$ such that $|\hat{\psi}(z)|<\lambda_{1}<1$ when $|z|<\delta_{1}$, and $|\hat{\psi}(z)|>\lambda_{2}>1$ when $|z|>1-\delta_{2}$. Set

$$
H_{1}=\operatorname{span}\left\{k_{z}:|z|<\delta_{1}\right\}
$$

and

$$
H_{2}=\operatorname{span}\left\{k_{z}:|z|>1-\delta_{2}\right\} .
$$

Thus $H_{1}$ and $H_{2}$ are dense subsets of $H$ (see Proposition 4.2 in [13]). Put $T=W_{\psi, \varphi}^{*}$. For every $z \in B_{N}$,

$$
T^{n}\left(k_{z}\right)=\left[\prod_{j=1}^{n-1} \overline{\hat{\psi}\left(\hat{\varphi}_{j}(z)\right)}\right] k_{\hat{\varphi}_{n}(z)} .
$$

If $|z|<\delta_{1}$, then $\left|\hat{\varphi}_{n}(z)\right|=|z|<\delta_{1}$ and for each positive integer $n,\left|\hat{\psi}\left(\varphi_{n}(z)\right)\right|<$ $\lambda_{1}<1$. And if $|z|>1-\delta_{2}$, then $\left|\hat{\varphi}_{n}(z)\right|>1-\delta_{2}$. Therefore the sequence $\left\{T^{n}\right\}$ converges pointwise to zero on the dense subset $H_{1}$. Define a sequence of linear maps $S_{n}: H_{2} \rightarrow H_{2}$ by

$$
S_{n} k_{z}=\left[\prod_{j=1}^{n-1} \overline{\left(\hat{\psi}\left(\hat{\varphi}_{j}^{-1}(z)\right)\right)^{-1}}\right] k_{\hat{\varphi}_{n}^{-1}(z)}
$$

for every $z \in B_{N}$. If $\left|z_{1}\right|>1-\delta_{2}$, then $S_{n} k_{z} \rightarrow 0$ as $k \rightarrow \infty$. Thus the sequence $\left\{S_{n}\right\}$ converges pointwise to zero on the dense subset $H_{2}$. Besides, $T^{n} S_{n} k_{z}=k_{z}$ on $H_{2}$, therefore $T$ satisfies the hypothesis of the hypercyclicity criterion and $T$ is hypercyclic.

Acknowledgment. The authors would like to thank the referee for carefully reading the paper and providing corrections and suggestions for improvements.

\section{References}

[1] F. Bayart, A class of linear fractional maps of the ball and their composition operators, Adv. Math. 209 (2007), no. 2, 649-665.

[2] C. Bisi and F. Bracci, Linear fractional maps of the unit ball: A geometric study, Adv. Math. 167 (2002), no. 2, 265-287.

[3] P. S. Bourdon and J. H. Shapiro, Cyclic composition operators on $H^{2}$, Operator theory: operator algebras and applications, Part 2 (Durham, NH, 1988), 43-53, Proc. Sympos. Pure Math., 51, Part 2, Amer. Math. Soc., Providence, RI, 1990.

[4] _ Cyclic phenomena for composition operators, Mem. Amer. Math. Soc. 125 (1997), no. 596, $\mathrm{x}+105 \mathrm{pp}$. 
[5] F. Bracci, M. D. Contreras, and S. Díaz-Madrigal, Classification of semigroups of linear fractional maps in the unit ball, Adv. Math. 208 (2007), no. 1, 318-350.

[6] K. C. Chan and J. H. Shapiro, The cyclic behavior of translation operators on Hilbert spaces of entire functions, Indiana Univ. Math. J. 40 (1991), no. 4, 1421-1449.

[7] X. Chen, G. Cao, and K. Guo, Inner functions and cyclic composition operators on $H^{2}\left(B_{n}\right)$, J. Math. Anal. Appl. 250 (2000), no. 2, 660-669.

[8] J. B. Conway, Function of One Complex Variable, Springer-Verlag, 1973.

[9] C. C. Cowen and B. D. MacCluer, Composition Operators on Spaces of Analytic Functions, CRC Press, Boca Roton, 1995.

[10] L Linear fractional maps of the ball and their composition operators, Acta Sci. Math. (Szeged) 66 (2000), no. 1-2, 351-376.

[11] N. S. Feldman, The dynamics of cohyponormal operators, Trends in Banach spaces and operator theory (Proc. Conf., Memphis, TN, 2001), 71-85.

[12] R. M. Gethner and J. H. Shapiro, Universal vectors for operators on spaces of holomorphic functions, Proc. Amer. Math. Soc. 100 (1987), no. 2, 281-288.

[13] G. Godefroy and J. H. Shapiro, Operators with dense invariant cyclic vector manifolds, J. Funct. Anal. 98 (1991), no. 2, 229-269.

[14] K. G. Grosse-Erdmann, Universal families and hypercyclic operators, Bull. Amer. Math. Soc. (N.S.) 36 (1999), no. 3, 345-381.

[15] _ Recent developments in hypercyclicity, Rev. R. Acad. Cien. Serie A. Mat. 97 (2003), no. 2, 273-286.

[16] L. Jiang and C. Ouyang, Cyclic behavior of linear fractional composition operators in the unit ball of $\mathbb{C}^{N}$, J. Math. Anal. Appl. 341 (2008), no. 1, 601-612.

[17] C. Kitai, Invariant closed sets for linear operators, Thesis, Univ. of Toronto, 1982

[18] B. D. MacCluer, Iterates of holomorphic self-maps of the unit ball in $\mathbb{C}^{N}$, Michigan Math. J. 30 (1983), no. 1, 97-106.

[19] S. Rolewicz, On orbits of elements, Studia Math. 32 (1969), 17-22.

[20] W. Rudin, Function Theory in the Unit Ball of $\mathbb{C}^{n}$, Grundlehren Math. Wiss., vol. 241, Springer-Verlag, New York, 1980.

[21] H. N. Salas, Hypercyclic weighted shifts, Trans. Amer. Math. Soc. 347 (1995), no. 3, 993-1004.

[22] B. Yousefi and H. Rezaei, Hypercyclic property of weighted composition operators, Proc. Amer. Math. Soc. 135 (2007), no. 10, 3263-3271.

[23] _ Some necessary and sufficient conditions for Hypercyclicity Criterion, Proc. Indian Acad. Sci. Math. Sci. 115 (2005), no. 2, 209-216.

[24] K. Zhu, Spaces of Holomorphic functions in the Unit Ball, Graduate Texts in Mathematics, 226. Springer-Verlag, New York, 2005.

REN-Yu CHEN

Department of Mathematics

Tianjin University

Tianjin 300072, P. R. China

E-mail address: chenry@tju.edu.cn

Ze-HuA ZHOU

Department of Mathematics

TIANJIN UNIVERSITY

Tianjin 300072, P. R. China

E-mail address: zehuazhou2003@yahoo.com.cn 